La Revue

des Droits

de l'Homme

\section{La Revue des droits de l'homme}

Revue du Centre de recherches et d'études sur les droits fondamentaux

9 | 2016

Revue des droits de l'homme - $\mathrm{N}^{\circ} 9$

\title{
L'absence de la précarité sociale parmi les motifs de discrimination reconnus par le droit français : un frein normatif à l'effectivité de la lutte contre les discriminations?
}

Ioannis Rodopoulos

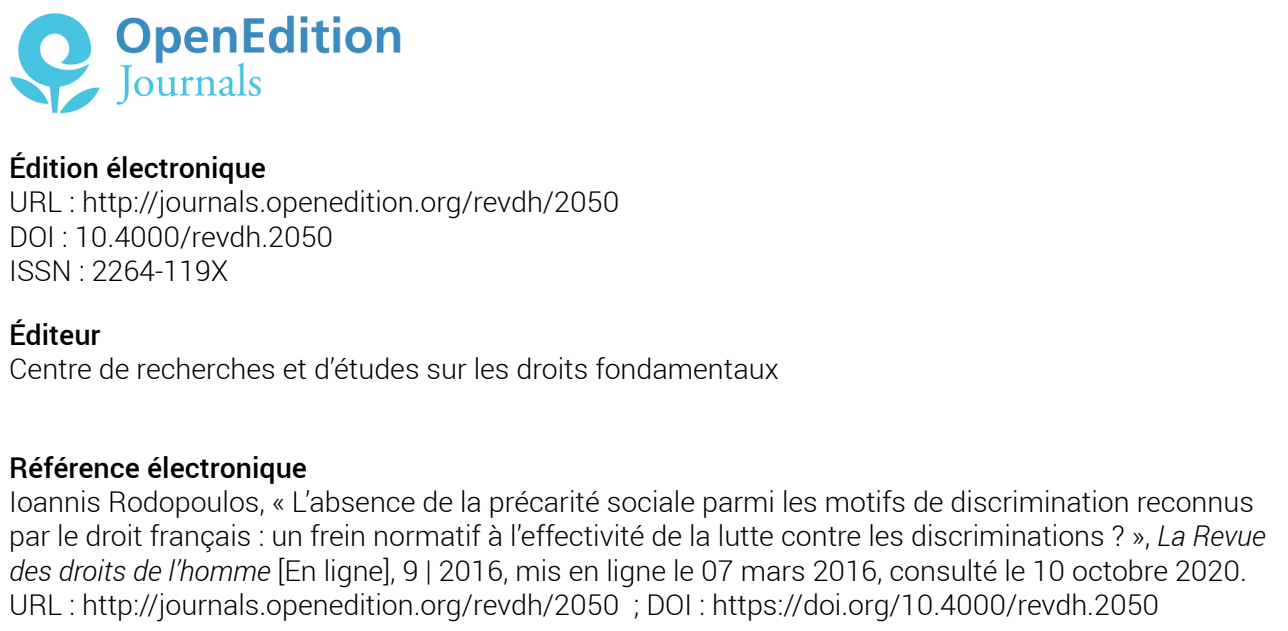

Ce document a été généré automatiquement le 10 octobre 2020

Tous droits réservés 


\title{
L'absence de la précarité sociale parmi les motifs de discrimination reconnus par le droit français : un frein normatif à l'effectivité de la lutte contre les discriminations?
}

\author{
Ioannis Rodopoulos
}

\section{De la nécessité d'une nouvelle notion juridique de discriminations fondées sur la précarité sociale}

1 La liaison entre précarité sociale et discriminations n'est guère novatrice. Depuis le travail pionnier de Lester Thurow ${ }^{1}$, les sociologues ont, à plusieurs reprises, souligné que la précarité conduit à la discrimination, tout comme la discrimination conduit à la précarité ${ }^{2}$ Moins présente dans le champ juridique, la question n'en est pour autant pas entièrement absente. Malgré la diversité des vocables utilisés, tant le droit international que certains droits nationaux ${ }^{3}$ interdisent depuis un certain temps les discriminations fondées sur des critères économiques.

Pour sa part, le droit français reste encore muet sur ce point. On observe, cependant, depuis quelques années - notamment, face aux nouvelles données posées par la crise financière - l'émergence d'une volonté d'étudier et de lutter contre ce type de discriminations. Ainsi le 26 septembre 2013, l'assemblée plénière de la Commission nationale consultative des droits de l'homme ( $\mathrm{CNCDH})$ adopta à l'unanimité un avis sur les discriminations fondées sur la précarité sociale ${ }^{4}$ proposant, entre autres, l'insertion d'un nouveau motif de discrimination dans l'article 225-1 du Code pénal ${ }^{5}$. Quelques jours plus tard, et dans le même esprit, un livre blanc fut publié par le Mouvement ATD (Agir Tous pour la Dignité) Quart Monde ${ }^{6}$. Clairement inspirée par les recommandations de la CNCDH et du Mouvement ADT Quart Monde, une proposition de loi « visant à 
lutter contre la discrimination à raison de la précarité sociale » fut déposée au Sénat le 31 mars 2015. Adoptée en première lecture par le Sénat le 18 juin 2015, cette proposition fut néanmoins modifiée selon les recommandations de la Commission des lois : à la place de la notion de précarité sociale, on voit désormais apparaitre celle de "particulière vulnérabilité résultant de sa situation économique, apparente ou connue de son auteur $»^{7}$.

3 Il est déjà manifeste que, si l'absence de la précarité sociale parmi les motifs de discrimination reconnus par le droit français pourrait constituer un frein normatif à l'effectivité de la lutte contre les discriminations, il y a également une série de freins conceptuels qui ont jusqu'ici empêché la reconnaissance de ce motif, tellement important mais, à la fois, tellement difficile à saisir. En effet, une certaine confusion est inévitable; non seulement en raison du signifié plurivoque et relatif de la notion de précarité sociale, mais aussi en raison des divers signifiants juridiques quasisynonymes qu'on voit apparaitre en alternance dans des textes nationaux, européens ou internationaux : fortune, origine sociale, situation économique et sociale, condition sociale, vulnérabilité ${ }^{8}$. Il est certes évident qu'il existe un noyau dur sémantique commun à toutes ces notions, mais il y a aussi des écarts importants. Par exemple, la notion de condition sociale comprend sans doute, mais n'est en aucun cas limitée, à la seule situation économique d'un individu. Or, même les notions de situation économique ou de fortune n'auraient pas tout à fait les mêmes conséquences juridiques que la notion de précarité. Par exemple, une discrimination fondée sur la fortune de quelqu'un est concevable sans que la personne discriminée soit nécessairement considérée comme socialement précaire.

4 Cela étant dit, ce n'est pas uniquement la notion de précarité sociale qui pose problème ; c'est aussi la nature de la discrimination elle-même qui manque de clarté par rapport à ce motif. Car, contrairement à d'autres motifs de discrimination usuels et au-delà des difficultés conceptuelles susmentionnées -, on pourrait soutenir - de manière un peu provocatrice - que les discriminations fondées sur la situation économique font partie inhérente du système socio-politique libéral (du moins dans sa conception actuelle). Il est par exemple sans doute injustifié et illégal - même si en pratique il n'est pas rare - de refuser de louer son appartement à quelqu'un en raison de sa couleur, de sa race, de son orientation sexuelle ou de sa religion, mais il est tout à fait conforme à la loi de refuser la location à quelqu'un qui ne présente pas certaines garanties de solvabilité. À plus forte raison, il est légitime de refuser de fournir une prestation à quelqu'un qui n'est pas en mesure d'en payer le prix. Certes, la problématique de la distinction entre discriminations justifiées et injustifiées n'est pas propre aux discriminations fondées sur la situation économique. Mais la situation économique présente une spécificité ontologique par rapport aux autres motifs de discrimination car le traitement différencié selon la situation économique de quelqu'un est très souvent toléré, sinon imposé par la loi, ce qui n'est pas le cas pour la plupart des autres motifs de discrimination, du moins pas au même degré. Cette spécificité rend la distinction entre les discriminations légitimes et illégitimes très complexe et incertaine.

5 Compte tenu de ces difficultés, le but de cet article est, dans un premier temps, d'examiner si la consécration d'une nouvelle notion juridique de discriminations fondées sur la précarité sociale paraît nécessaire afin de rendre le droit français, d'une part, conforme aux obligations internationales qui engagent la France, d'autre part, 
efficace dans sa lutte contre les discriminations (I). Dans un deuxième temps, il s'agira de s'interroger sur la voie qu'il faudra suivre afin de combler les lacunes éventuelles du droit français, sans sacrifier ses principes fondamentaux et sa cohérence structurelle (II).

6 L'évaluation de la nécessité de l'ajout d'une nouvelle catégorie aux motifs de discrimination déjà reconnus par le législateur présuppose des considérations tant d'ordre normatif (A) que d'ordre politique (B).

\section{A. Une nécessité normative}

7 Du point de vue normatif, il faudra tout d'abord étudier les obligations internationales et européennes qui contraignent le législateur français, puis l'état actuel du droit français, afin d'éviter des redondances et des conflits de normes.

\section{1) Les sources internationale et européennes}

8 En ce qui concerne les sources internationales et européennes, le constat est celui d'une proportion inverse : moins contraignants sont les textes juridiques, plus poussée est leur référence aux discriminations fondées sur la précarité sociale. Les raisons sautent aux yeux : il est facile de souligner l'importance du problème des discriminations antipauvres, mais il est très difficile de traduire cette importance en normes juridiques applicables et opérationnelles.

9 Les pas les plus importants vers la reconnaissance des discriminations économiques ont été faits dans le cadre du droit des Nations Unies. Plus précisément, l'interdiction des discriminations fondées sur la fortune et l'origine sociale apparaît déjà dans l'article 2 de la Déclaration universelle des droits de l'homme de $1948^{\circ}$ : "Chacun peut se prévaloir de tous les droits et de toutes les libertés proclamés dans la présente Déclaration, sans distinction aucune, notamment de race, de couleur, de sexe, de langue, de religion, d'opinion politique ou de toute autre opinion, d'origine nationale ou sociale, de fortune, de naissance ou de toute autre situation ". La même formule a été reprise dans d'autres textes onusiens, notamment dans le Pacte international relatif aux droits civils et politiques ${ }^{10}$ et le Pacte international relatif aux droits économiques, sociaux et culturels.

10 Les notions de fortune et d'origine sociale ont été davantage précisées par le Comité onusien des droits économiques, sociaux et culturels (CODESC) ${ }^{11}$, selon lequel « l'origine sociale renvoie à la position sociale héréditaire d'une personne ». Quant à la fortune, il s'agit, selon le Comité, « d'une notion vaste qui comprend les biens immobiliers (par ex., la propriété ou l'occupation de terres) et les biens personnels (par ex., la propriété intellectuelle, les biens mobiliers et les revenus) ou leur absence ». La notion de fortune est donc interprétée par le Comité d'une manière neutre. L'interdiction des discriminations fondées sur la fortune ne concerne pas uniquement les pauvres, mais aussi les riches.

11 Cela étant dit, à côté des motifs de discrimination expressément interdits par le Pacte, les experts onusiens en ajoutent d'autres, qui concernent des « formes de traitement différencié qui n'ont pas de justification raisonnable et objective et sont comparables aux motifs que le paragraphe 2 de l'article 2 cite expressément $»^{12}$. Parmi ces motifs, on voit apparaitre «la situation économique et sociale ", à propos de laquelle le Comité 
précise: «Des individus et des groupes de population ne doivent pas être traités arbitrairement en raison de leur appartenance à une certaine catégorie économique ou sociale ou à une certaine couche sociale. La situation sociale et économique d'une personne pauvre ou sans domicile fixe, peut faire qu'elle est constamment en butte à une discrimination, à une stigmatisation et à des stéréotypes négatifs, ce qui peut avoir pour conséquence qu'on lui refuse la même qualité d'éducation et de soins de santé que les autres ou le même accès à ces services, ou qu'on lui refuse l'accès aux lieux publics ou le même accès que les autres ${ }^{13}$ On voit ainsi apparaître un nouveau critère, celui de la situation économique et sociale qui, malgré sa parenté sémantique avec les critères précédents d'origine sociale et de fortune, a une connotation légèrement différente et renvoie clairement à ce que l'on entend par précarité sociale. Il est par ailleurs intéressant, que l'observation du CoDESC ne se contente pas de préciser les motifs de discrimination, mais donne des exemples concrets, à titre indicatif bien entendu, des formes que pourrait prendre une telle discrimination.

Enfin, plus récemment, un rapport intitulé Principes directeurs sur l'extrême pauvreté et les droits de l'homme, soumis le 18 juillet 2012 par Mme Magdalena Sepúlveda Carmona en application de la résolution $17 / 13$ du Conseil des droits de l'homme ${ }^{14}$, témoigne de l'importance croissante que prennent pour les institutions onusiennes tant les discriminations fondées sur la précarité que la précarité elle-même. Selon les recommandations contenues dans ce rapport, les États sont invités à prendre «toutes les mesures nécessaires pour éliminer toute discrimination directe ou indirecte à l'encontre des personnes vivant dans la pauvreté », à « s'abstenir d'adopter toute loi ou tout règlement ou pratique susceptible de priver les personnes vivant dans la pauvreté de l'accès à la jouissance de tous leurs droits ", à « examiner la législation nationale afin de repérer tout impact discriminatoire sur ceux qui vivent dans la pauvreté ", à « abroger ou modifier toute loi qui a pour objectif ou conséquence de compromettre la jouissance égale des droits par ceux qui vivent dans la pauvreté ", ainsi qu'à adopter "une législation exhaustive sur la lutte contre la discrimination à l'égard des personnes vivant dans la pauvreté » qui puisse être « appliquée par les tribunaux ». Ces orientations générales sont concrétisées par une série de suggestions plus spécifiques, qui relèvent de natures et de logiques diverses. On y trouve des mesures à caractère pédagogique ${ }^{15}$, des mesures préventives et répressives ${ }^{16}$, des mesures pénales et nonpénales ${ }^{17}$, des mesures substantielles ${ }^{18}$ et processuelles ${ }^{19}$, qui ont toutes pour but commun d'assurer une protection solide et complète des droits civiques ${ }^{20}$, civils ${ }^{21}$ et sociaux $^{22}$, positifs ${ }^{23}$ et négatifs ${ }^{24}$, des personnes vivant dans la pauvreté.

En ce qui concerne le droit du Conseil de l'Europe, une formule presque identique à celle de la Déclaration universelle des droits de l'homme a été insérée dans la Convention européenne des droits de l'homme (article 14), puis dans le Protocole $\mathrm{n}^{\circ} 12$ de la même Convention: la jouissance des droits et libertés prévus doit être assurée "sans distinction aucune, fondée notamment sur le sexe, la race, la couleur, la langue, la religion, les opinions politiques ou toutes autres opinions, l'origine nationale ou sociale, l'appartenance à une minorité nationale, la fortune, la naissance ou toute autre situation ".

14 Toutefois, la jurisprudence de la Cour européenne des droits de l'homme concernant les discriminations économiques est pratiquement inexistante. Le seul arrêt qui condamne une discrimination fondée sur la fortune est l'arrêt Chassagnou du 29 avril 199925. Mais dans cet arrêt les requérants étaient des petits propriétaires terriens qui étaient 
discriminés par rapport à de grands propriétaires. Peu importe quelle définition de la précarité, absolue ou relative, on adoptera, il paraît discutable de parler de précarité ou d'extrême pauvreté dans le cas de petits propriétaires terriens. L'affaire Chassagnou est donc un excellent exemple du fait que, malgré leur parenté, les notions de fortune et de précarité sociale ne visent pas les mêmes réalités. Certes, les notions de précarité et de pauvreté sont englobées par les catégories plus générales d'origine sociale et de fortune. Mais elles représentent un phénomène social spécifique, qui comporte des particularités bien distinctes et qui justifieraient une reconnaissance juridique explicite et autonome.

Enfin, le droit de l'Union européenne apparaît quant à lui encore plus silencieux sur cette question. La fortune et l'origine sociale ne constituent pas des motifs de discrimination explicitement reconnus, ce qui s'explique partiellement par la logique un peu différente du législateur européen, qui n'a pas cherché à donner une définition générale des discriminations, mais plutôt à interdire les discriminations, directes ou indirectes, fondées sur des motifs spécifiques : la race et l'origine ethnique ${ }^{26}$, la religion et les croyances, le handicap, l'âge, l'orientation sexuelle ${ }^{27}$. Cela étant dit, l'absence de l'origine sociale et de la fortune parmi les motifs reconnus par les directives - et surtout par la directive 2000/78/CE - ne semble guère justifiée, soit qu'il s'agisse d'une omission volontaire ou involontaire du législateur européen. Tout d'abord, la directive, engageant les États membres quant à l'objectif à atteindre en leur laissant le choix des moyens et de la forme pour atteindre cet objectif, serait l'instrument idéal pour responsabiliser les États, sans poser des problèmes sérieux au regard des particularités des droits nationaux. Par ailleurs, cette absence est d'autant plus injustifiée que l'on trouve dans les textes des références abstraites à la cohésion ou l'insertion économique et sociale ou même à la solidaritée ${ }^{28}$; des références qui reflètent cependant simplement des buts politiques, sans avoir aucune portée normative directe.

\section{2) Les sources nationales}

16 Comme il a été déjà dit, en droit français, il n'y a pour l'instant aucune protection juridique explicite et générale contre les discriminations fondées sur la précarité sociale. Certaines dispositions ponctuelles existent, telle la procédure prévue par l'article L.1110-3 du Code de la santé publique permettant de sanctionner des refus de soins opposés aux bénéficiaires de la Couverture maladie universelle. Mais, comme le fait observer Diane Roman, «le dispositif demeure peu appliqué et les droits souvent théoriques $»^{29}$.

17 Bien entendu, les études sociologiques en la matière démontrent que les discriminations fondées sur la précarité n'apparaissent guère isolées, mais sont le plus souvent intrinsèquement liées à d'autres attitudes discriminatoires, relatives à l'appartenance ethnique, religieuse, sociale ou sexuelle de la victime, à son lieu de résidence, etc. ${ }^{30}$ On pourrait donc soutenir qu'en réalité les cas de discrimination qui restent à l'abri du droit français sont finalement peut-être plus limités qu'on ne l'imagine. Il n'en reste pas moins qu'il y a - ou qu'il peut y avoir - des discriminations fondées exclusivement sur la précarité et, à cet égard, le droit français présente effectivement une lacune peu justifiée.

18 Il reste à voir si l'interdiction des discriminations fondées sur la précarité sociale correspond également à une nécessité politique. 


\section{B Une nécessité politique}

19 Si l'on parle d'une nécessité politique, il ne s'agit pas ici d'examiner le degré de consensus autour de l'adoption d'une nouvelle loi ou de procéder à une nébuleuse casuistique interprétant abstraitement les valeurs de liberté, égalité et fraternité - cela dépasserait largement les ambitions de cet article -, mais de voir, d'un point de vue stratégique, dans quelle mesure une nouvelle notion juridique serait nécessaire afin de mettre en œuvre les objectifs officiellement déclarés des autorités politiques actuelles.

Selon le rapport Kaltenbach sur la proposition de loi du 31 mars $2015^{31}$, l'objectif de la nouvelle loi serait double : faire reculer la discrimination systémique et apporter une reconnaissance symbolique à un problème social souvent caché. Plus précisément, selon le rapport, «la pauvreté est ressentie comme une double peine: en sus de la précarité matérielle, cette situation se double d'une stigmatisation. Or, le sentiment d'humiliation entretient les phénomènes de discrimination. Selon les témoignages des associations, les humiliations ressenties sont d'une telle violence que ces personnes préfèrent en effet ne pas demander les prestations auxquelles elles ont droit, de peur d'être stigmatisées [...] Plus que l'introduction d'un critère manquant à notre législation, les associations qui luttent contre la pauvreté et l'exclusion sociale demandent un engagement de la République sur ces questions ».

21 La question qui se pose est donc de voir si ces deux objectifs, pour légitimes qu'ils soient, justifient l'adoption d'une nouvelle loi. La réponse est a priori oui, avec cependant quelques réserves, tant par rapport à l'effectivité potentielle d'une nouvelle loi, que par rapport à son impact symbolique.

S'il est indéniable qu'il y a une atteinte portée à l'égalité quand on discrimine quelqu'un parce qu'il est pauvre, l'atteinte la plus importante à l'égalité ne résulte pas ici de l'acte discriminatoire en soi, mais de la situation de pauvreté elle-même. Certes, d'un point de vue juridique, il est nécessaire de classifier séparément les deux questions. La ratio legis derrière l'interdiction des discriminations fondées sur la précarité sociale est de lutter plutôt contre les discriminations que contre la pauvreté. Toutefois, contrairement à d'autres formes de discriminations, cette lutte aura peu à offrir si elle n'est pas accompagnée de mesures luttant contre la précarité elle-même ${ }^{32}$.

Par ailleurs, même la reconnaissance symbolique du problème pourrait avoir des effets pervers. Car la plupart des autres motifs de discrimination - y compris la fortune - sont conçus sur des notions axiologiquement neutres. L'interdiction des autres formes de discriminations peut plus ou moins se résumer dans l'impératif de traiter de manière égale toute personne, indépendamment de sa nationalité, de sa couleur, de sa religion, de ses orientations sexuelles, etc. Or, la précarité - tout comme le handicap - n'est pas une situation neutre. Elle est un problème majeur en soi. Il faut donc distinguer entre acceptation ou protection des personnes en précarité et normalisation de leur situation. Autrement dit, il ne faut pas que la reconnaissance symbolique des discriminations fondées sur la précarité serve d'alibi pour ne pas lutter contre la précarité elle-même, même par voie de mesures de "discrimination positive " ${ }^{33}$, telles que celles préconisées par les rapports susmentionnés des Nations Unies ou du Conseil de l'Europe. Par ailleurs, la précarité n'étant pas, comme certains autres motifs de discrimination, une qualité figée de l'individu, mais une situation ouverte qui se redéfinit sans cesse - en théorie, toute personne peut se trouver à tout moment dans une situation de précarité et, de la même manière, peut en sortir -, la « discrimination 
positive » en matière de précarité ne présente pas les mêmes inconvénients que celle en matière, par exemple, de race ou de sexe (traitement inégal, stigmatisation, etc.) ; son but n'est pas tout simplement de protéger la personne dans sa situation, mais de l'aider à sortir de cette situation. En réalité, la « discrimination positive » en faveur des personnes en situation de précarité n'est rien de plus et rien de moins que ce qu'on a appelé depuis plus d'un siècle la manifestation d'un «État providence ».

Acceptant, malgré ces réserves, les avantages, voire la nécessité de l'introduction dans la loi d'un nouveau motif de discrimination, la question se pose alors des modalités de traduction de cette volonté politique en norme juridique opérationnelle.

\section{Les voies de concrétisation d'une notion juridique de discriminations fondées sur la précarité sociale}

Comme le rappelle le rapport Kaltenbach, si le droit pénal a longtemps été en droit français la voie privilégiée pour lutter contre les discriminations, la question s'est, depuis, particulièrement développée en droit social, en droit civil, en droit économique $^{34}$. Fidèle à cette tradition, la proposition de loi déposée au Sénat comporte des mesures concernant tant le champ pénal que le non pénal. En effet, si la voie pénale présente des avantages considérables, tant pour l'effectivité du droit que pour la sécurité juridique, elle a aussi ses limites (A), ce qui rend nécessaire le recours complémentaire à d'autres branches du droit (B).

\section{A. Les avantages et les limites d'un recours au droit pénal}

La question du choix entre criminalisation et régulation d'un problème en dehors du champ pénal n'est certes pas propre aux discriminations fondées sur la précarité sociale. Elle relève d'une problématique plus générale qui implique la prise en compte de facteurs d'ordre juridique et politique tels que l'importance de l'intérêt juridique protégé, la nature de l'atteinte à cet intérêt ou la gravité de cette atteinte. Cela étant dit, la question des discriminations fondées sur la précarité présente certaines particularités qui méritent quelques remarques plus spécifiques.

\section{1).L'opportunité du choix de la voie pénale}

Les avantages du recours à la répression pénale sont à la fois substantiels et processuels. Sur le plan substantiel, outre le fait que les sanctions pénales sont en général considérées, par leurs gravités, plus effectives et dissuasives que les sanctions civiles ou administratives - ce qui peut être parfois discutable ${ }^{35}-$, le grand avantage ici est de nature symbolique et pédagogique. En effet, bien que les discriminations contre les personnes en situation de précarité soient clairement incompatibles avec les principes fondamentaux du droit, elles sont en pratique souvent tolérées, voire justifiées par une partie de la population, sur la base d'une perception déformée de la liberté contractuelle, économique, etc. Contrairement à une simple interdiction - dans le cadre, par exemple, du droit du travail -, l'incrimination du comportement visé symboliserait, comme pour les autres types de discriminations prohibés par la loi pénale, une désapprobation de la société face à un acte socialement et moralement intolérable. Pour emprunter un concept assez contestable du point de vue théorique, 
mais bien illustratif de la différence symbolique entre les deux types de sanctions, par son incrimination, la discrimination fondée sur la précarité sociale ne sera pas un acte simplement interdit (malum prohibitum), mais deviendra un acte moralement inacceptable (malum in se), qui sera stigmatisé en tant que tel par la société. Si l'on accepte que les discriminations injustifiées portent atteinte à des valeurs fondamentales de la société dans son ensemble, une telle consécration symbolique semble être légitime.

Or, les avantages du recours à la voie pénale ne concernent pas uniquement le droit substantiel, mais aussi le droit processuel. En effet, nonobstant les garanties renforcées relatives aux droits de la défense, il y a des cas où le droit pénal peut être plus effectif aussi dans la protection des intérêts des victimes de discriminations, notamment quand ces victimes se trouvent en situation de précarité sociale. Tout d'abord, la charge de la preuve de la partie plaignante se reporte sur le ministère public, ce qui peut favoriser, dans certains cas, l'accès à la justice de victimes de discriminations, qui auraient des difficultés à prouver leurs assertions. Par ailleurs, depuis le 11 juillet 2007, il existe dans chaque parquet un pôle anti-discrimination qui comprend un magistrat référent et un délégué du procureur de la République ${ }^{36}$. Enfin, selon la Cour de cassation, le juge pénal est libre de prendre en compte tout moyen de preuve, y compris des preuves obtenues de manière illicite ou déloyale $e^{37}$, ce qui n'est pas le cas en procès civil. Pour les personnes précaires, qui n'ont souvent pas accès à une assistance juridique digne de ce nom, la possibilité d'une collecte de preuves d'office par le ministère public est un avantage indéniable.

\section{2) Les exigences liées au choix de la voie pénale}

Toutefois, pour que des sanctions pénales soient appliquées, il faut qu'une série de conditions soient remplies. Le principe de la légalité des délits et des peines exige d'une part que la loi pénale soit claire et précise (lex certa), d'autre part qu'elle soit écrite (lex scripta). Une incrimination générale des discriminations fondées sur la précarité sociale, combinant une notion déjà problématique quant à la clarté de la loi - celle de discrimination - avec une notion extrêmement floue, flexible et jamais définie par la loi - celle de précarité sociale - aurait comme conséquence de laisser la tâche de définir le comportement pénalement sanctionné au juge, ce qui ne pourrait pas être acceptable à l'aune des principes fondamentaux du droit pénal. Par ailleurs, un regard sur la jurisprudence du Conseil constitutionnel à propos de l'inceste et du harcèlement sexuel ${ }^{38}$ suggère qu'une telle incrimination aurait peu de chances de survivre à une saisine des juges de la rue Montpensier. Le droit de ne pas être discriminé en raison de sa précarité sociale ressemble plutôt à un bien juridique qui devrait être protégé par le législateur pénal par des formes plus précises (par exemple, par la voie de l'aggravation d'autres infractions) qu'à un énoncé d'incrimination autonome et opérationnel.

Afin de surmonter cet obstacle, la Commission des lois du Sénat opta pour la substitution à la notion de précarité sociale de celle de "vulnérabilité résultant de la situation économique de la personne ", une notion qui, sans être synonyme de la précarité, peut couvrir plus ou moins les mêmes situations en matière de discriminations. Ce choix doit être félicité puisqu'il constitue un pas de concrétisation de la proposition précédente assez important: non pas tellement parce que la vulnérabilité est en soi une notion beaucoup plus précise que la précarité sociale, mais parce qu'elle constitue déjà une notion juridique traitée depuis un certain temps par la 
jurisprudence et par la doctrine. Toutefois, il ne faut pas oublier que jusqu'à aujourd'hui, la vulnérabilité apparait, en droit pénal, associée à des comportements plus concrets ${ }^{39}$. Ce qui est puni est en fait l'exploitation de la vulnérabilité de quelqu'un, afin d'obtenir un profit matériel, sexuel, etc. L'exploitation de la vulnérabilité est une notion plus précise - et plus facilement prouvable - qu'une simple discrimination en raison de la vulnérabilité. Ainsi, même si l'on peut accepter que la notion de vulnérabilité soit préférable comme motif de discrimination pénalement sanctionné à la notion de précarité sociale, il n'est pas certain que la nouvelle formule soit absolument conforme à la Constitution et aux principes fondamentaux du droit.

\section{B. La nécessité d'un recours complémentaire à des mesures non pénales}

31 Indépendamment des avantages que pourrait offrir une réponse pénale aux discriminations fondées sur la précarité, sa portée sera inévitablement limitée. Elle devra reposer sur des règles spécifiques, interprétées de manière stricte et appliquées de manière conforme aux droits de la défense (notamment au droit à la présomption d'innocence). Ces exigences ne sont certes pas spécifiques au motif de la précarité sociale. Elles concernent la politique pénale contre les discriminations dans son ensemble. Or, pour les raisons susmentionnées, liées à la spécificité ontologique de la précarité comme motif de discrimination, tant l'élément matériel (distinction entre discrimination justifiée et injustifiée) que l'élément moral (intention spécifique de discriminer quelqu'un parce qu'il est pauvre) d'une infraction de discrimination fondée sur la précarité peuvent s'avérer très difficiles à prouver, surtout quand il s'agit de discriminations indirectes. Par conséquent, pour que la lutte contre les discriminations des personnes en situation de précarité soit effective, il sera indispensable d'adopter des règles additionnelles, tant en droit privé (droit civil, droit social, droit commercial, etc.), qu'en droit administratif. Comme dans le cas du droit pénal, les avantages du recours aux autres branches juridiques sont substantiels, liés notamment à la possibilité de définitions plus souples, mais également processuels, liés notamment aux différentes modalités de la preuve ${ }^{40}$.

Plus précisément, comme il a été déjà dit, une des difficultés les plus importantes concernant l'incrimination des discriminations fondées sur la précarité est la distinction entre discriminations justifiées et injustifiées. Cette difficulté n'est l'apanage ni des discriminations fondées sur la précarité, ni du choix de la voie pénale. Dans tous les cas de discrimination, et même dans les branches juridiques non pénales, une telle distinction peut s'avérer pour le juge un exercice casuistique délicat et complexe, puisqu'il faudra trouver un équilibre entre des valeurs et des droits subjectifs contradictoires.

Toutefois, la question de la précarité présente deux spécificités importantes. La première est que, si, pour les autres motifs, la justification d'un acte discriminatoire peut s'appuyer sur des critères externes au motif de discrimination interdit, dans le cas de la précarité, il y a des hypothèses où la discrimination pourrait être légitimée par le critère de la situation économique lui-même (pour reprendre l'exemple donné plus haut, dans le cas du refus de location d'un logement à un candidat locataire nonsolvable). La deuxième spécificité est que la précarité est une situation - sociale et personnelle - très complexe, qui en comporte d'autres. Pour fournir une illustration, en 
s'inspirant de l'affaire bien médiatisée d'une famille en grande précarité qui a été expulsée du musée d'Orsay au motif que leur odeur indisposait les autres visiteurs ${ }^{41}$, on peut imaginer des cas similaires, où le responsable d'un acte discriminatoire pourrait défendre sa décision sur des critères liés, à titre d'exemple, à la santé publique. Dans un tel cas, il serait quasiment impossible de prouver le degré de culpabilité requis pour imposer une sanction pénale, alors qu'il semble moins ardu de constater une faute civile ou un acte illicite de l'administration. Dans de tels cas, c'est uniquement par une évolution jurisprudentielle lente - et éventuellement controversée - que le droit pourra trouver au cours des années l'équilibre nécessaire entre liberté (économique, d'expression, etc.) et droit à la non-discrimination. Et une telle évolution ne saurait avoir lieu en matière pénale, où cette tâche est censée être exclusivement réservée au législateur.

Or, le recours au droit non pénal présente aussi des avantages du point de vue processuel, notamment au regard de l'administration de la preuve, nonobstant les difficultés posées par le fait qu'en droit civil il appartient aux parties d'apporter les preuves. Tout d'abord, comme il a été déjà dit, la preuve absolue d'une discrimination intentionnelle étant parfois très difficile à obtenir, la présomption d'innocence peut constituer, en droit pénal, un frein normatif très important - et en même temps indispensable - à l'effectivité de l'interdiction des discriminations, surtout quand les discriminations sont fondées sur un motif aussi complexe que la précarité sociale. Certes, on peut s'interroger sur le fait de savoir si des personnes en situation de précarité seront en mesure de revendiquer leurs droits devant les juridictions civiles ou administratives. Mais il y a des solutions face à de tels problèmes, des solutions qui ne peuvent être acceptables qu'en dehors du procès pénal. L'aménagement de la charge de la preuve apportée par l'article 4 de la loi du 27 mai 2008 en est un exemple caractéristique.

Au moment où, en Europe comme ailleurs, «l'art d'ignorer les pauvres $»^{42}$ ne cesse de se sophistiquer, la reconnaissance par le législateur d'une situation sociale existante voire en pleine croissance - et sa volonté de lutter contre cette situation, doivent être saluées. Les difficultés techniques de cette démarche sont nombreuses; les dangers pour la sécurité juridique aussi. Or, toute innovation juridique est un pas vers l'inconnu, comportant des difficultés, des risques d'abus, de l'incertitude. Au lieu de renoncer à l'effort courageux de lutter contre un phénomène doublement inhumain, la tâche du juriste devra être d'assurer que cette lutte se réalise d'une manière normativement et conceptuellement cohérente : tout d'abord lors de l'élaboration du droit, mais aussi - et surtout - lors de son application. À défaut d'une telle cohérence, non seulement le problème ciblé ne sera pas résolu, mais la situation des personnes discriminées de fait risquera de se détériorer. Le manque de sécurité juridique ne joue jamais - du moins pas à long terme - en faveur des personnes vulnérables. 


\section{NOTES}

1. Cf. inter alia, KATZ Michael B., The Undeserving Poor, 2nd edition, Oxford University Press, New York, 2013 ; LANG Kevin Poverty and Discrimination, Princeton University Press, Princeton NJ, 2007 ; MUNGER Frank (ed.), Law and Poverty, Ashgate, Aldershot, UK, 2006

2. Cf. inter alia, KATZ Michael B., The Undeserving Poor, 2nd edition, Oxford University Press, New York, 2013 ; LANG Kevin Poverty and Discrimination, Princeton University Press, Princeton NJ, 2007 ; MUNGER Frank (ed.), Law and Poverty, Ashgate, Aldershot, UK, 2006

3. On peut citer comme exemples les droits belge, canadien, bolivien, sud-africain, équatorien. Pour plus de détails, $c f$. notamment l'Étude de législation comparée du Sénat, $n^{\circ} 253$, février 2015, Les sanctions applicables à la discrimination à raison de la pauvreté, réalisée à la demande de $\mathrm{M}$. Yannick VAUGRENARD, Sénateur de la Loire- Atlantique. Sur le droit canadien, $c f$. notamment ROMAN Diane, «La discrimination fondée sur la condition sociale, une catégorie manquante du droit français ", Recueil Dalloz, 2013, p. 1911.

4. J.O.R.F. du 9 octobre 2013, texte no 40, NOR : CDHX1324396V. Sur ce texte, cf. également ROMAN Diane, «La discrimination fondée sur la précarité sociale, un nouvel outil en faveur de l'effectivité des droits ? ", Lettre " Actualités Droits-Libertés » du CREDOF, 8 octobre 2013.

5. Discrimination et pauvreté. Livre blanc: analyse, testings et recommandations, Octobre 2013. Une étude menée par ATD Quart Monde France, Institut de recherche et de formation aux relations humaines et ISM Corum.

6. Rapport $n^{\circ} 507$ (2014-2015) de M. Philippe KALTENBACH, fait au nom de la commission des lois constitutionnelles, de législation, du suffrage universel, du Règlement et d'administration générale sur la proposition de loi de M. Yannick VAUGRENARD et plusieurs de ses collègues visant à lutter contre la discrimination à raison de la précarité sociale, déposé au Sénat le 10 juin 2015.

7. Comme on le verra par la suite (IA et IB), les notions de fortune et d'origine sociale apparaissent dans la Charte universelle des droits de l'homme, dans divers textes onusiens, ainsi que dans la Convention européenne des droits de l'homme; la notion de situation économique et sociale a été évoquée par le Comité onusien des droits économiques, sociaux et culturels ( $c f$. infra, note no 13); la notion de condition sociale apparaît notamment dans le droit canadien (cf. ROMAN Diane, op. cit., passim) ; enfin, comme il a été déjà mentionné, la notion de vulnérabilité résultant de sa situation économique a été proposée ( $c f$. note précédente) pour remplacer la notion de précarité sociale dans la proposition de loi susmentionnée du 31 mars 2015.

8. Cf. cependant à cet égard la décision du Tribunal de 1ère instance de Namur, du 5 mai 2015, qui a, pour la première fois en Belgique, constaté l'existence d'une discrimination sur base de la fortune dans la mise en location d'un logement (Trib.1ière instance Namur, 5-05-2015, R.G. 305/15, Rép. 3938, ordre 15).

9. Art. $2 \$ 1,24 \S 1$ et 26 Nations Unies, Pacte international relatif aux droits civils et politiques (16 décembre 1966), A/RES/2200 (XXI).

10. Art. $2 \S 1,24 \S 1$ et 26 Nations Unies, Pacte international relatif aux droits civils et politiques (16 décembre 1966), A/RES/2200 (XXI).

11. Document NU E/C.12/GC/20 du 2 juillet 2009, Observation générale no 20 : La non-discrimination dans l'exercice des droits économiques, sociaux et culturels (art. 2, par. 2 du Pacte international relatif aux droits économiques, sociaux et culturels), notamment $\$ \$ 24,25$ et 35

12. Idem, $\$ 27$

13. Id., $\$ 35$

14. Principes directeurs sur l'extrême pauvreté et les droits de l'homme, présentés par la rapporteuse spéciale sur les droits de l'homme et l'extrême pauvreté, Madame Sepulveda Carmona, le 18 
juillet 2012 (A/HRC/21/39), et adoptés par consensus le 27 septembre 2012 par le Conseil des droits de l'homme de l'ONU (A/HRC/RES/21/11).

15. «Les États doivent prendre des mesures spéciales pour protéger ceux qui vivent dans la pauvreté contre la violation de leurs droits par des tiers. À cette fin, ils doivent : i) Réaliser des programmes éducatifs et des campagnes pour sensibiliser la population aux nombreux obstacles auxquels se heurtent les personnes vivant dans la pauvreté pour surmonter leur situation ; ii) Encourager les médias à éviter les informations partisanes et les couvertures sensationnelles qui perpétuent les stéréotypes discriminatoires à l'égard des personnes vivant dans la pauvreté. Pour ce faire, les États devraient promouvoir un journalisme éthique et encourager l'adoption de codes de conduite pour éliminer l'image négative des personnes vivant dans la pauvreté, des sans-abri, des chômeurs et des personnes recevant des prestations sociales ", op. cit.

16. «Les États devront adopter des mesures législatives pour empêcher et punir les violations des droits des personnes vivant dans la pauvreté par des entités privées ", op. cit.

17. «Les États doivent assurer une aide juridique de qualité aux secteurs les plus pauvres de la société, non seulement dans le cadre de procédures pénales, mais aussi en ce qui concerne les questions intéressant particulièrement les personnes vivant dans la pauvreté, notamment les appels relatifs aux prestations sociales et les procédures d'expulsion et de protection de l'enfant », op. cit.

18. «Les lois qui ciblent spécifiquement les comportements et actions des personnes vivant dans la pauvreté relèvent de la discrimination sur la base du statut économique et social et doivent être abrogées ", op. cit.

19. «Les États doivent faire en sorte que toutes les politiques pénales et règlementaires soient conformes aux normes relatives aux droits de l'homme, y compris les principes d'égalité et de non-discrimination et la présomption d'innocence ». "L'accès à une représentation juridique revêt une importance primordiale et s'applique à toutes les formes de pénalisation utilisées à l'encontre des personnes vivant dans la pauvreté ». Les États doivent "veiller, dans toute la mesure possible, à ce que les procédures de libération sous caution tiennent compte de la situation économique et sociale des personnes vivant dans la pauvreté », op. cit.

20. Les États « doivent repérer et écarter les obstacles institutionnels qui empêchent les groupes vulnérables et marginalisés de participer pleinement aux processus de prise de décisions ", op. cit. 21. «Les politiques de surveillance, les conditions imposées et autres exigences doivent être révisées de sorte qu'elles ne violent pas les obligations relatives aux droits de l'homme en imposant un fardeau disproportionné à ceux qui vivent dans la pauvreté. Durant la collecte et le traitement des informations concernant les bénéficiaires, les États doivent veiller à ce que les normes internationalement acceptées en matière de vie privée et de confidentialité soient respectées et que ces informations ne soient pas communiquées à d'autres autorités ou utilisées à d'autres fins sans le consentement des bénéficiaires ", op. cit.

22. «Les États doivent créer un environnement qui favorise la participation des personnes vivant dans la pauvreté à la vie publique et aux décisions qui touchent à leur vie », op. cit.

23. «Les États doivent créer un environnement qui favorise la participation des personnes vivant dans la pauvreté à la vie publique et aux décisions qui touchent à leur vie », op. cit.

24. «Les États doivent examiner toutes les politiques et législations relatives à la détention et à l'incarcération afin d'y repérer et d'en retirer les lois et les pratiques discriminatoires qui ont des incidences disproportionnées sur les personnes vivant dans la pauvreté. Des mesures devraient être mises en place pour permettre à la police, aux tribunaux et aux fonctionnaires publics d'évaluer convenablement les effets que peut avoir la détention et l'incarcération, compte tenu de la situation personnelle de chacun " "Les États ne doivent avoir recours à la détention et à l'incarcération que pour répondre à un besoin sociétal urgent et d'une manière proportionnelle à ce besoin. Ils doivent veiller à ce que l'arrestation ou la détention n'ait pas d'incidences disproportionnées sur les personnes vivant dans la pauvreté ». 
25. Chassagnou et autres c. France, réq. 25088/94, 28331/95 et 28443/95, CEDH, 29 avril 1999

26. Directive 2000/43/CE du Conseil du 29 juin 2000 relative à la mise en œuvre du principe de l'égalité de traitement entre les personnes sans distinction de race ou d'origine ethnique, J.O. L 180 du 19.7.2000, p. 22.

27. Directive 2000/78/CE du Conseil du 27 novembre 2000 portant création d'un cadre général en faveur de l'égalité de traitement en matière d'emploi et de travail, J.O. L 303 du 2.12.2000, p. 16

28. Directive 2000/78/CE, cons. 11 ; Directive 2000/43/CE, cons. 9.

29. Op. cit.

30. $C f$. inter alia MEYER BISCH Patrice, « Discriminations multiples et interdépendance entre les violations des droits de l'homme. Essai sur l'humiliation », in ZUFFEREY Jean-Baptiste, DUBEY Jacques, PREVITALI Adriano (éds.), L'homme et son droit. Mélanges en l'honneur de Marco Borghi, Schulthess, Zürich, 2011, pp. 331-348. cf. aussi H. Kombila, «Les entraves à l'approche « intersectionnelle » canadienne de la discrimination », cette revue, infra.

31. Op. cit., p. 15

32. Pour des analyses plus détaillées sur cette question, $c f$. notamment Conseil de l'Europe, Vivre en dignité au XXIe siècle, Pauvreté et inégalité dans les sociétés de droits humains : le paradoxe des démocraties, Éditions du Conseil de l'Europe, 2013.

33. Du moins si l'on abandonne le postulat libéral selon lequel toute personne serait responsable de sa situation économique, et si l'on admet que, surtout en temps de crise économique, il est souvent impossible de sortir de la précarité par ses propres moyens

34. Rapport Kaltenbach, op. cit., p. 11 et 14.

35. En effet, il est souvent soutenu que, dans plusieurs cas, les sanctions civiles ou administratives - en principe moins graves mais plus faciles à appliquer - peuvent être plus effectives que les sanctions pénales. Cette idée, guère nouvelle, a déjà été préconisée par Beccaria : " la perspective d'un châtiment modéré, mais auquel on est sûr de ne pouvoir échapper, fera toujours une impression plus vive que la crainte vague d'un supplice terrible, dont l'espoir de l'impunité anéantit presque toute l'horreur " (Des délits et des peines, Institut Coppé, Paris, 2011, chapitre XVII). Bien entendu, la problématique générale du choix entre sanctions pénales et non pénales dépasse largement les ambitions de cet article.

36. Cf. rapport Kaltenbach, op. cit., p. 14.

37. Crim, 11 juin 2002, cité dans le rapport Kaltenbach, op. cit., p. 14.

38. Cf. Cons. Const. $\mathrm{n}^{\circ} 2011-163$ QPC du 16 septembre 2011 ; Cons. Const. n 2012-222 QPC du 17 février 2012 ; Cons. Const. n 2012-240 QPC du 4 mai 2012.

39. Les articles 225-13 et 225-14 du Code pénal incriminent respectivement «le fait d'obtenir d'une personne dont la vulnérabilité ou l'état de dépendance sont apparents ou connus de leur auteur, la fourniture de services non rétribués ou en échange d'une rétribution manifestement sans rapport avec l'importance du travail accompli» et le fait de soumettre celle-ci «à des conditions de travail ou d'hébergement incompatibles avec la dignité humaine ». Par ailleurs, depuis la loi $n^{\circ} 2012-954$ du 6 août 2012 relative au harcèlement sexuel, ce dernier est aggravé lorsque les faits sont commis sur "une personne dont la particulière vulnérabilité ou dépendance résultant de la précarité de sa situation économique ou sociale est apparente ou connue de son auteur ».

40. Par ailleurs, la reconnaissance de ces avantages n'est pas une nouveauté. $C f$., à titre d'exemple, la loi no 2008-496 du 27 mai 2008 portant diverses dispositions d'adaptation au droit communautaire dans le domaine de la lutte contre les discriminations (JORF no $0123 \mathrm{du} 28$ mai 2008, p. 8801), avec laquelle le législateur introduisit la notion de discrimination indirecte, imposée par le droit communautaire.

41. Le 26 janvier 2013, un couple et son fils adolescent qui visitaient le musée d'Orsay, accompagnés d'un membre d'ATD Quart-Monde, ont été priés de quitter les lieux en raison de leur odeur. Pour ATD Quart-Monde, la famille a été victime d'une discrimination liée à sa 
situation de pauvreté. L'affaire, qui a fait sensation dans la presse, est aussi mentionnée dans l'avis précité de la CNCDH, p. 1.

42. Cf. GALBRAITH John Kenneth, "L'art d'ignorer les pauvres ", Le Monde Diplomatique, octobre 2005, p. 6 (républié de Harper's Magazine, Nov. 1985).

\section{RÉSUMÉS}

Existant depuis longtemps en droit international, ainsi que dans un certain nombre de droits étrangers - même sous des vocables divers -, la notion de discriminations fondées sur la précarité sociale demeure à ce jour pratiquement absente du droit français. Afin de combler cette lacune, une proposition de loi fut déposée au Sénat le 31 mars 2015, proposant une série de mesures, y compris l'insertion d'un nouveau critère de discrimination dans l'article 225-1 du Code pénal. Les débats relatifs à ce texte ainsi que l'étude de la littérature sociologique en la matière démontrent que l'intérêt et l'actualité de la question ne sauraient être contestées; sa complexité non plus: complexité normative, certes, mais aussi complexité conceptuelle, complexité des valeurs que la loi doit protéger, complexité du contexte socio-historique actuel. L'objet de cet article est dans un premier temps d'examiner si l'absence de la précarité sociale parmi les critères de discrimination reconnus par le droit français constitue un frein normatif à l'effectivité de la lutte contre les discriminations afin de mesurer le degré de nécessité d'une nouvelle loi. Dans un deuxième temps, seront formulées quelques réflexions relatives à la forme que cette nouvelle loi, à la supposer utile, pourrait prendre.

The notion of discrimination on the grounds of social precariousness exists since quite some time - although under different names - in international law, as well as in some domestic legal systems. It is still, however, not present in French law. In order for this gap to be filled, a law proposal has been submitted to the Senate on the 31st of March 2015, including, amongst other measures, the introduction of a new motive of discrimination into Article 225-1 of the Penal Code. A quick study of this proposal - the legislative procedure is still ongoing -, as well as of the sociological literature on the field, is convincing enough on the importance and the topicality of the question; on its complexity as well though : a complexity which is not only normative, but also conceptual, a complexity of protected values, a complexity of the current socio-historical context. The aim of this article is firstly to examine if the absence of social precariousness amongst the recognised motives of discrimination can be a normative obstacle to the effectiveness of the fight against discrimination and to which extent a new law would be necessary. Secondly, the aim is to come up with some thoughts on the form that the new law could take.

\section{INDEX}

Mots-clés : lutte contre les discriminations, précarité sociale, pauvreté, situation économique, droit pénal.

Keywords : fight against discrimination, social precariousness, poverty, economic situation, criminal law. 


\section{AUTEUR}

\section{IOANNIS RODOPOULOS}

Ioannis Rodopoulos est docteur en droit -chercheur postdoctoral à l'Université du Luxembourg 